\title{
PREDICADOS PSICOLÓGICOS Y ESTRUCTURAS CON VERBO LIGERO: DEL ESTADO AL EVENTO*
} PSYCH PREDICATES AND LIGHT VERB CONSTRUCTIONS: FROM STATE TO EVENT

\author{
RICARD VIÑAS-DE-PUIG \\ East Carolina University \\ vinasdepuigr@ecu.edu
}

\section{RESUMEN}

En este artículo se explora desde una perspectiva generativa el comportamiento en castellano y catalán de los predicados psicológicos que resultan de construcciones con verbo ligero y se analiza su estructura eventiva. Se parte de una Estructura Experimentadora BÁsICA, en la que el argumento EXPERIENCIA es una raíz unida a $V$, que introduce la FuENTE (o Tema) de la experiencia. Por su parte, el Experimentador es un argumento externo, introducido por la proyección funcional $v_{\text {EXP }}$. Esta Estructura EXPERIMENTADORA Básica sólo puede ofrecer una lectura estativa; las lecturas eventivas, si se dan, son el resultado de una combinación de factores: i. la unión a la estructura de las proyecciones $v_{\text {вECOME }} \mathrm{y}$, opcionalmente, $v_{\text {caus }}$; ii. la concordancia de los rasgos eventivos de estas proyecciones funcionales con los que vienen subcategorizados en la raíz EXPERIENCIA. En caso de que se una la proyección $v_{\text {BECOME }}$ al $S v_{\text {EXP }}$ (y haya concordancia de rasgos), se obtiene una lectura incoativa; en cambio, si se une el núcleo $v_{\text {caus }}$ (y haya concordancia de rasgos), que introduce un CAUSANTE, la interpretación será la de un predicado causativo. Este análisis, además, da cuenta de la variación que se observa tanto en catalán como en castellano de la variación de la forma fonológica del verbo ligero.

Palabras clave: Verbos psicológicos, estructura argumental, estructura eventiva, verbos ligeros.

\footnotetext{
* Este trabajo fue financiado en parte por el proyecto NCS-0345680 de la National Science Foundation: "Mayangna: A database and study of nominal features on its verbal inflection system". Asimismo, quisiera agradecer a los asistentes al 13th Hispanic Linguistics Symposium y al 21st Colloquium on Generative Grammar sus comentarios y sugerencias a versiones anteriores de este artículo.
} 


\begin{abstract}
In this article I explore, within the generative framework, the behavior of psych predicates resulting from light verb constructions in both Spanish and Catalan, paying special attention to their eventive structure. I initially propose a BASIC EXPERIEnCE STRUCTURE, whereby the Experience is an independent root merged on a $\mathrm{V}$ head, which is responsible for the introduction of the Source (or Theme) of the experience. The Experiencer, on the other hand, is an external argument introduced by the functional projection $v_{\text {ExP }}$. This BAsic EXPERIENCE Structure only yields stative readings; the eventive interpretations, if they surface, are the result of combining two factors: i. merging the functional projections $v_{\text {весоме }}$ and, optionally, $v_{\text {СAus }}$; and ii. establishing a matching operation of the eventive features of these functional projections with those subcategorized in the EXPERIENCE root. If the unaccusative projection $v_{\text {весомв }}$ is merged (and feature matching takes place), an inchoative reading is obtained; if the projection $v_{\text {CAUS }}$, which introduces a CAUSER at an external argument position, is merged (and feature matching takes place), the reading will be that of a causative experience predicate. Besides, this analysis accounts for the variation in the phonological output of the light verb, observed in both Catalan and Spanish.
\end{abstract}

Keywords: Psych verbs, argument structure, eventive structure, light verbs.

Recibido: 21.10.2013. Aceptado: 14.07.2014.

\title{
1. INTRODUCCIÓN
}

\subsection{La cuestión}

- $\mathrm{n}$ este artículo, desde una perspectiva generativista, se analiza el comportaCmiento de los predicados experimentadores con verbo ligero (PEVL) tanto en castellano como en catalán. A partir de una estructura experimentadora básica se propone que los PEVL en su configuración básica son estados; las interpretaciones eventivas, si se dan, son el resultado de una suma de condicionantes: i. se añaden proyecciones funcionales eventivas a la estructura estativa básica; y ii. estas proyecciones funcionales concuerdan con los rasgos eventivos que presenta el argumento EXPERIENCIA. Por lo tanto, en la propuesta que aquí se presenta se combina un análisis de subcategorización (relativo a los rasgos eventivos presentes en el argumento cuando entra en la derivación sintáctica) con un análisis constructivista (con la introducción de proyecciones funcionales que establecen una relación sintáctica con el argumento EXPERIENCIA). Además, este análisis también sirve para explicar la variación observada en la forma del verbo ligero, en concordancia con otros trabajos recientes sobre predicados experimentadores (cf. Crous y Gràcia, 2013).

Aunque en la bibliografía se observan varios análisis que dan cuenta (desde diferentes puntos de vista teóricos) de la estructura argumental y la interpretación 
eventiva de los predicados experimentadores (o verbos psicológicos, como a menudo se denominan) (Pesetsky, 1987, 1995; Belletti y Rizzi, 1988; Rigau, 1990; Bouchard, 1992; Masullo, 1992; Croft, 1993; Torrego, 1996; Arad, 1998, 1999; Haspelmath y Caruana, 2000; McGinnis, 2000, 2001; Rosselló, 2002; Pylkkänen, 2002, 2008; Cuervo, 2003; Landau, 2005; Adger y Ramchand, 2006; Marín y McNally, 2009; entre muchos otros), bastante menos atención se ha prestado a los PEVL (cf. Cuervo, 2010; Viñas-de-Puig, 2009; y otros más). Algunos ejemplos de estos tipos de construcciones se dan en castellano, (1) catalán (2) y mayangna (3) (todos los ejemplos en mayangna usados en este artículo proceden del trabajo de Charles y Torrez (2008)).

(1) Me dan asco las ratas.

(2) Em fa mal la mà. 'Me duele la mano.'

$\begin{array}{lll}\text { (3) } & \text { Tingki dalâ } & \text { yâwi. }^{1} \\ \text { mano-Conc.1sG daño } & \text { DAT.1sG-[dar]-Prs.3sG }\end{array}$ 'Me duele la mano.'

En todos los ejemplos anteriores, el predicado contiene un verbo ligero, con variación dependiendo de la lengua: dar en castellano (1), fer 'hacer' en catalán (2) y yâwi 'dar' en mayangna (3). Cabe señalar, además, que en estos tres ejemplos, la interpretación que se obtiene es la de un estado (es decir, una lectura no eventiva). Sin embargo, como sucede también con otros tipos de predicados experimentadores, existe la posibilidad de obtener una lectura eventiva. Tal variación en la interpretación se observa en los ejemplos en catalán que se muestran en (4):

(4) (a) Em fan por els comentaris racistes.

'Los comentarios racistas me asustan.'

(b) Aquells nens em van fer por ahir.

'Esos niños me asustaron ayer.'

En los dos ejemplos en (4) se observa un contraste en la interpretación: en (4a) la lectura que se obtiene es la de un estado, sin un cambio en el evento o un iniciador de la experiencia descrita; en (4b), por el contrario, la primera lectura es la de un evento, con un obvio iniciador (o causante) de la experiencia. No obstante, esta variación en la lectura eventiva no es posible para todos estos tipos de predicados. Véanse las diferencias entre los pares en (5) y (6), para castellano y catalán

${ }^{1}$ En este artículo se usan las abreviaturas siguientes: 1: $1^{\text {a }}$ persona; 2: $2^{\text {a }}$ persona; 3: $3^{\text {a }}$ persona; Ac: Acusativo; C: Complementante; Conc: Marcador de concordancia; D: Determinante; DAT: Dativo; Inf: Infinitivo; Neg: Negación; Nom: Nominativo; PL: Plural; Prs: Presente; Psd: Pasado; ReFL: Reflexivo; SG: Singular. 
respectivamente, con los dos presentados en (4):

(5) (a) Me dan asco las ratas.

(b) \#Esos niños me dieron asco ayer.

(6) (a) Em fan fastic les rates. 'Me dan asco las ratas.'

(b) "Aquells nens em van fer fastic ahir. 'Esos niños me dieron asco ayer.'

A diferencia de lo observado en (4), los dos pares de frases en (5) y (6) no presentan una variación en la lectura eventiva: tanto en (5a) como en (5b) la interpretación que se obtiene es la de un estado; lo mismo ocurre con los ejemplos en (6a) y (6b). Esta lectura estativa se mantiene incluso con la introducción de un argumento animado (esos niños en (5b), aquells nens en (6b)) que podría favorecer una interpretación agentiva y/o causativa.

\subsection{Propuesta y antecedentes}

Con el objetivo de explicar esta variación, en este trabajo se propone que la interpretación eventiva de los PEVL en castellano y catalán depende de dos factores interrelacionados: i. los rasgos eventivos que se expresan (por subcategorización) en el argumento EXPERIENCIA (véase la Sección 2 para observar la presencia independiente de este constituyente); y ii. estos rasgos entran en una relación de ligamiento con los rasgos que se expresan en cada representación de la proyección funcional (recursiva) $v$ (en sus diferentes 'modalidades' $v_{\text {EXP }}, v_{\text {BECOME }}$ у $v_{\text {CAUS }}$ ).

Los predicados experimentadores han sido objeto de extensa investigación (Pesetsky, 1987, 1995; Belletti y Rizzi, 1988; Rigau, 1990; Bouchard, 1992; Masullo, 1992; Croft, 1993; Torrego, 1996; Arad, 1998, 1999; Haspelmath y Caruana, 2000; McGinnis, 2000, 2001; Rosselló, 2002; Pylkkänen, 2002, 2008; Cuervo, 2003, 2010; Landau, 2005; Adger y Ramchand, 2006; Marín y McNally, 2009; entre muchos otros). Tomando como base la clasificación ya clásica que aparece en Belletti y Rizzi (1988), más recientemente Fábregas, Marín y McNally (2012) presentan una ordenación actualizada de estos predicados. En la categorización que se observa en (7), los predicados de experiencia se clasifican según el Caso y el papel temático que recibe el EXPERIMENTADOR (Fábregas et al., 2012: 164):

(7) (a) Experimentador sujeto: querer, admirar, odiar, temer.

(b) Experimentador dativo: gustar, interesar, molestar, preocupar.

(c) Experimentador acusativo: molestar, preocupar, espantar.

(d) Experimentador en forma se: preocuparse, aburrirse, enfadarse, asustarse. 
Sin embargo, esta clasificación no incluye todos los casos de predicados experimentadores observados en varias lenguas, ya que no incluye los predicados formados con verbo ligero. Como ya se ha indicado, algunos autores (Viñas-de-Puig, 2009; Cuervo, 2010) dan muestra de la presencia de PEVL. Estos predicados, en los que el argumento EXPERIENCIA aparece de forma independiente, se observan en varias lenguas (no necesariamente relacionadas), como demuestran los ejemplos (1) a (3) anteriores.

La variación en la lectura eventiva es otro asunto importante en la realización de los predicados experimentadores. Dentro de la bibliografía generativista, empezando por la obra de Belletti y Rizzi (1988), se observan varios trabajos en los que se indica que algunos predicados experimentadores pueden ofrecer tanto interpretaciones estativas como eventivas. En la Sección 3 se discute dicha variación, argumentando que la variabilidad interpretativa está relacionada no solo con la presencia de proyecciones funcionales eventivas unidas a la estructura por encima de $S v_{\text {EXP }}$, sino que también tiene que establecerse una relación de ligamiento entre los rasgos de los núcleos funcionales eventivos y los que vienen subcategorizados en el argumento EXPERIENCIA.

\subsection{Organización del trabajo}

El presente artículo está divido de la forma siguiente. En la Sección 2 se presenta la propuesta de la existencia de una estructura universal de predicados experimentadores y sus diferentes posibilidades estructurales. La Sección 3, que es la parte principal del trabajo, se centra en dar cuenta de la variación en la interpretación que se obtienen con los PEVL, incluyendo una breve discusión sobre el tipo de estado que se obtiene. En esta misma sección se presentan las lecturas eventivas de dichos predicados, mostrando evidencia de su existencia y dando cuenta del análisis que se propone. Por último, en la Sección 4 se hace un resumen de los puntos principales del trabajo y se presentan algunas cuestiones que quedan abiertas para investigaciones futuras.

\section{UNA PROPUESTA BÁSICA}

Antes de analizar la interpretación eventiva de los PEVL, se hace necesario analizar la estructura argumental de estas construcciones. En esta sección se presentan evidencias a favor de la existencia de una estructura básica de predicados experimentadores, que da cuenta de la presencia tanto del verbo ligero como de los demás argumentos. 


\subsection{Una estructura universal para los predicados experimentadores}

Como ya se ha indicado en la sección anterior, la clasificación de predicados experimentadores que se presenta en Fábregas et al. (2012), basada en otras observadas en la bibliografía (ej. Belletti y Rizzi, 1988; Pesetsky, 1995; entre muchos otros), no es del todo exhaustiva. Cuervo (2010) y Viñas-de-Puig (2009) presentan evidencias de la existencia de los PEVL. Puesto que en estas construcciones (en su interpretación básica), el EXPERIMENTADOR aparece en caso Dativo, el autor considera que estos predicados forman parte de la categoría presentada en (7b) que se propone en Fábregas et al. (2012).

A partir de estos análisis más recientes, se propone una estructura básica que nos sirve para explicar la existencia, en diferentes lenguas, de los PEVL y de otros predicados con verbo 'completo'. Esta Estructura Experimentadora Básica se presenta en (8):

(8) Estructura Experimentadora Básica

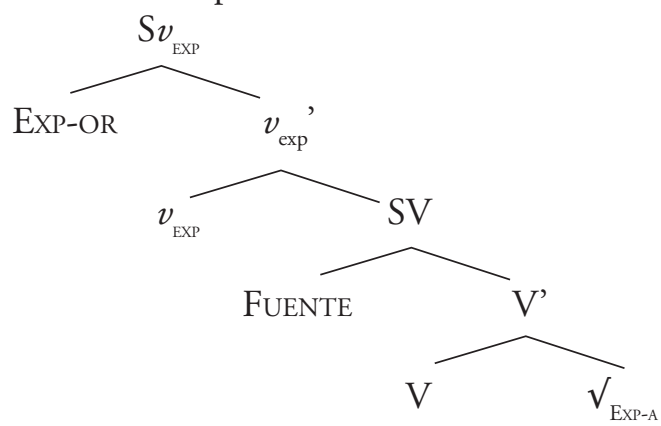

Como podemos comprobar en (8), la ExPERIENCIA $\left(V_{\text {Exp-A }}\right)$ es una raíz que se une al núcleo $\mathrm{V}$, creando así una estructura predicativa con el argumento FUENTE (llamado también TEMA por otros autores), introducido en el especificador del SV. Por otro lado, y de acuerdo con numerosos análisis generativistas sobre la introducción de argumentos externos (Kratzer, 1996; Arad, 1999; McGinnis, 2000; Pylkkänen, 2002, 2008; Cuervo, 2010; entre otros), el EXPERIMENTADOR (EXP-OR) viene introducido por la proyección $v_{\mathrm{EXP}}$.

Es lógico suponer entonces que este análisis nos ofrece dos posibilidades en relación a la expresión del argumento EXPERIENCIA (y la posible presencia de un verbo ligero). En la subsección que sigue, se presentan esas dos posibilidades y se ofrecen evidencias a favor de esta hipótesis.

\subsection{Experiencias incorporadas y experiencias independientes}

Si se observa la estructura presentada en (8), se puede ver que el argumento Ex- 
PERIENCIA está en posición adyacente al núcleo $\mathrm{V}$, lo que permite que se pueda incorporar a ese núcleo. Dicha posibilidad es el resultado de la estructura siguiente:

(9)

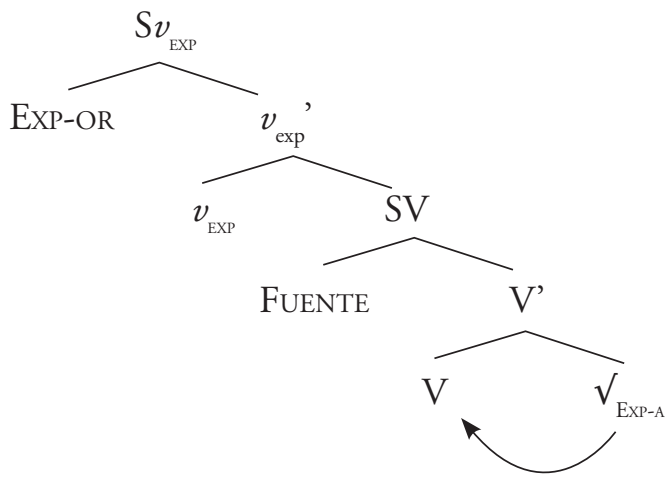

En (9), el argumento EXPERIENCIA aparece incorporado a V, con lo que se obtiene una construcción con un verbo completo (es decir, sin un verbo ligero). Se observan ejemplos de esta estructura en los predicados experimentadores en lenguas romances, como en castellano (10) o en catalán (11), pero también en lenguas de otras familias lingüísticas, como en mayangna (12) (lengua de la familia misumalpa) o inglés (13):

(10) Me duelen los brazos.

(11) Em molesten aquests comentaris.

'Me molestan estos comentarios.'

(12) Pedro kul kau sak

Pedro escuela en ser-Prs.3sg C

[... minikpa yak kal buihna ojo-COnc.3s en DAT.3sg temblar-Prs.3SG

'Cuando Pedro está en clase, le tiembla el ojo.'

(lit. 'Cuando Pedro está en clase, le tiembla en el ojo.')

(13) Those comments bothered me.

'Me molestaron esos comentarios.'

En todos estos ejemplos se observa cómo la EXPERIENCIA no aparece de forma independiente, sino que está incorporada en el núcleo V, y por lo tanto se comporta como un verbo léxico. Se puede advertir evidencias de su comportamiento verbal al ver las diferentes marcas de concordancia y tiempo: en castellano, duelen (10), en catalán molesten 'molestan' (11), en mayangna buihna 'tiembla' (12), y en inglés hurts 'duele' o bothered 'molestaron' (13).

Antes de seguir con la otra posibilidad relativa a la expresión del argumento EXPERIENCIA, hay que hacer hincapié en un aspecto importante del proceso que se 
describe en esta subsección. El proceso presentado en (9) y que da como resultado los ejemplos de (10) a (13) debe entenderse como un proceso de incorporación y no como un proceso de conflación. Siguiendo la propuesta de Mateu (2012), sólo nos encontraremos ante un proceso de conflación "in those constructions that involve Talmy's [...] conflation pattern, that is, the one that involves conflation of \{causation/motion\} with a subordinate supporting event" ("en aquellas construcciones que conllevan el patrón de conflación de Talmy [...], es decir, aquella que supone la conflación de \{causalidad/movimiento\} con un evento subordinado secundario" (Mateu, 2012: 255; mi traducción). Puesto que en los predicados resultantes de la estructura en (9) sólo se tiene un único evento, sin subeventos secundarios, se puede argumentar que el proceso por el que la raíz EXPERIENCIA $\left(V_{\text {Exp-A }}\right)$ se une al núcleo $\mathrm{V}$ es un proceso de incorporación, similar al descrito por Mateu (2012) (y referencias anteriores) o Hale y Keyser (1993, 2002), entre otros autores.

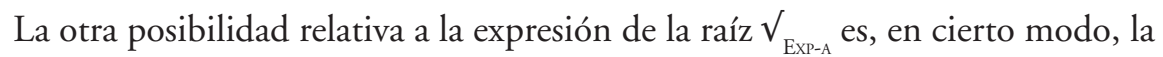
contraria. Partiendo de la misma estructura presentada en (9), se puede concebir

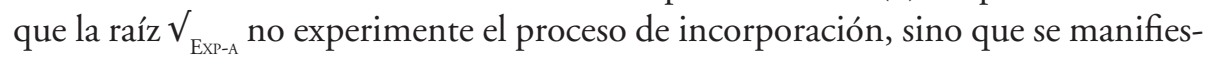
te de forma independiente, como se observa en (14):

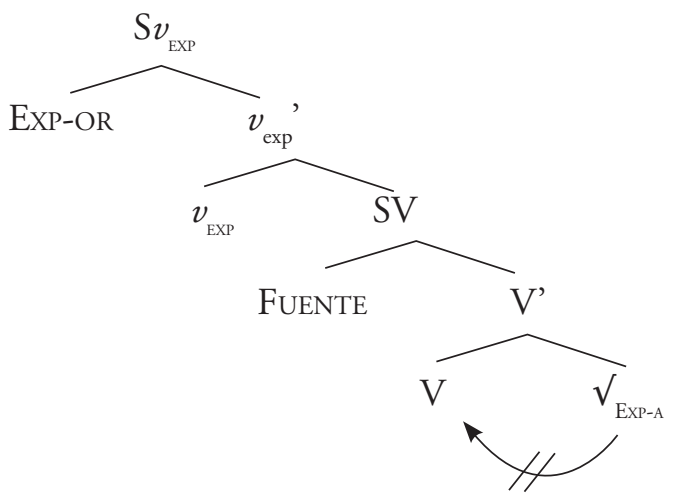

Como resultado de esta estructura, la presencia independiente de la raíz $\sqrt{E x p-A}_{\text {. }}$ conlleva la manifestación de un verbo ligero. Se observan expresiones de esta opción en lenguas romances, como lo demuestran los ejemplos en castellano y catalán en (15) y (16), así como en la familia misumalpa, como se observa en el ejemplo (17):

(15) (a) Me da miedo la oscuridad.

(b) Me dan asco las ratas.

(16) (a) Em fa mal la mà.

'Me duele la mano.' 
(b) Em fan por les serps. 'Me asustan las serpientes.'

(17) (a) Yuh kalawi.

hambre DAT.3sG-[dar]-Prs.3sG

'Tiene hambre.' (lit. 'Le da hambre.')

(b) Alasna yâwi.

felicidad DAT.1sG-[dar]-PRs.3SG

'Soy feliz.' (lit. 'Me da felicidad.')

En todos estos ejemplos, la raíz $\sqrt{\text { Exp-A }}_{\text {se }}$ se manifiesta de forma independiente al núcleo V. Y como se ha dicho, este hecho supone la presencia de un verbo ligero, cuya forma varía entre las diferentes lenguas analizadas (tanto en castellano como en mayangna se usa la forma correspondiente al verbo 'dar'; en catalán, por el contrario, se usa 'hacer').

Es importante destacar que la raíz no incorporada como resultado de la estructura presentada en (14) se comporta como un argumento sintácticamente independiente. $\mathrm{Al}$ igual que otros constituyentes independientes, este argumento EXPERIENCIA se puede cuantificar, como se observa en los ejemplos (18) y (19), en castellano y catalán respectivamente:

(18) Me da mucho miedo la oscuridad.

(19) Em fan molt mal els ulls.

'Me duelen mucho los ojos.'

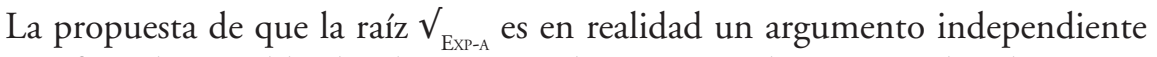
viene reforzada por el hecho de que este elemento puede ser reemplazado por un clítico partitivo. Este hecho es observable en catalán, como se demuestra en (20):

(20) No me'n $\mathbf{n}_{\mathrm{i}}$ fan gens __ els ulls, (de mal).

'No me duelen nada los ojos.'

En lo que a los dos argumentos de la estructura se refiere, la FuENTE y el ExPERIMENTADOR, éstos se unen a la estructura en dos niveles estructurales distintos y, por lo tanto, muestran propiedades diferentes. De acuerdo con varios análisis relativos a la introducción de argumentos externos, se propone que el EXPERIMENTADOR es un argumento externo (Belletti y Rizzi, 1988; Cuervo, 2003, 2010; Adger y Ramchand, 2006; Ramchand, 2008; cf. Masullo, 1992), que viene introducido por la proyección funcional $v_{\text {Exp }}$ (cf. Kratzer, 1996; Arad, 1999; McGinnis, 2000; Pylkkänen, 2002, 2008). Desde esta posición de argumento externo, el EXPERIMENTADOR puede establecer una relación de ligamiento con un argumento interno, como se observa en el ejemplo en catalán en (21): 
(21) $\mathbf{E m}_{\mathrm{i}}$ fan mal els $s_{\mathrm{i}}$ ulls. 'Me duelen los ojos.'

En (21), el EXPERIMENTADOR (expresado como clítico dativo em 'me') establece una relación de ligamiento con la FuENTE (el ulls 'los ojos'). Se obtiene así una interpretación (parasítica) de posesión: el argumento els ulls sólo puede ser poseído por la persona expresada por el EXPERIMENTADOR Dativo.

Con esta estructura completa, se observa que los PEVL conforman una subclase dentro de la clasificación que aportaban Fábregas et al. (2012): estos predicados pertenecen al grupo de verbos experimentadores con un EXPERIMENTADOR Dativo (7b), pero a diferencia de los demás predicados, incluyen la presencia independiente de la raíz $\sqrt{E x p-A}_{\text {. }}$.

Es importante señalar también que el análisis que se presenta en esta sección concuerda con las ideas sobre la configuración argumental de los predicados con EXPERIMENTAdor Dativo que se apunta en Marín y Sánchez Marco (2012). Si partimos de la base que los predicados que se analizan en este trabajo pertenecen a la clase de verbos psicológicos de experimentador objeto (VPEO) (es decir, el experimentador no es el sujeto de la oración, como en el caso de verbos como temer u odiar), el análisis construccionista que se deriva de la ESTRUCTURA EXPERIMENTADORA BÁSICA concuerda con lo establecido por estos autores: en su estudio del tipo de estatividad de los verbos y nombres psicológicos en castellano, Marín y Sánchez Marco (2012: 92) afirman que "el significado del verbo incluye el del nombre. Ello parece especialmente evidente en el caso de los VPEO, ya que muchos de estos verbos son denominales". Así pues, la estructura argumental que se propone en esta sección da cuenta de la posibilidad de que el nombre psicológico pueda aparecer incorporado al núcleo verbal (9) o de forma independiente (13), pero manteniendo siempre los mismos rasgos semánticos y eventivos. Esta idea será importante para analizar las lecturas eventivas de estos predicados, objeto de la Sección 3, en la que se presta especial atención a los PEVL que permiten una variación entre lecturas estativas y eventivas.

\section{VARIACIÓN EN LA INTERPRETACIÓN EVENTIVA}

Como ya se ha indicado, y siguiendo postulados de otros autores, algunos predicados experimentadores pueden mostrar lecturas tanto estativas como eventivas (Belletti y Rizzi, 1988; Pesetsky, 1995; Arad, 1999; McGinnis, 2001; Pylkkänen, 2002, 2008; Franco y Huidobro, 2003; Viñas-de-Puig, 2009; entre muchos otros):

(22) (a) Me asustan los comentarios racistas. 
Predicados psicológicos y estructuras con verbo ligero: Del estado al evento / R. VIÑAS-DE-PUIG

(b) Esos niños me asustaron ayer.

(23) (a) Em fan por els comentaris racistes. 'Me asustan los comentarios racistas.'

(b) Aquells nens em van fer por ahir. 'Esos niños me asustaron ayer.'

(24) (a) Racist comments scare me. 'Me asustan los comentarios racistas.'

(b) Those children scared me yesterday. 'Esos niños me asustaron ayer.'

En los ejemplos anteriores se puede observar el contraste interpretativo entre las frases (a) y (b): tanto en castellano como en catalán o en inglés, la frase (a) en estos ejemplos indica una experiencia estativa, sin que se indique un cambio de estado o un causante voluntario del mismo; en las frases (b), por el contrario, existe un causante de la experiencia (esos niños en (22b), aquells nens (en 23b) y those children (en 24b)), con lo que se obtiene una lectura eventiva causativa, con cambio de estado.

\subsection{Lecturas estativas}

Si nos centramos en la lectura de los PEVL tanto en castellano (25) como en catalán (26), nos daremos cuenta de que todos estos predicados pueden aparecer en construcciones con lecturas estativas:

(25) (a) Me dan miedo las ratas.

(b) Las arañas, ¿te dan asco?

(c) El pomelo le da dentera.

(d) ¿Por qué me da envidia la felicidad de los otros?

(e) Me da pavor conducir de noche.

(f) ¡Me da mucho gusto conocerla!

(g) Nos da mucha pena que se vayan sin comer.

(h) A Elvira, le dan lástima los mendigos de la calle.

(i) De pequeño, me daba vergüenza hablar con las niñas de la clase.

(j) ¡Me dan ganas de besarte!

(26) (a) Em fa mal la mà. 'Me duele la mano.'

(b) Li fan fastic les rates.

'Las ratas le dan asco.'

(c) Et fan por les pelis de terror?

‘'Te dan miedo las películas de terror?' 
(d) Ens fa molta angúnia veure això. 'Me da mucha angustia ver eso.'

(e) Em fa angoixa dormir amb la porta oberta.

'Me da zozobra dormir con la puerta abierta.'

Todos los ejemplos en (25) y en (26) se obtienen a partir de una estructura en la que la EXPERIENCIA es un argumento independiente y, por lo tanto, aparece un verbo ligero. En todos estos casos, además, la lectura que se obtiene es la de un estado. Esta noción es extensible a todos los PEVL y, por lo tanto, podemos concluir que todos los PEVL permiten una interpretación estativa. Teniendo este hecho en cuenta, y considerando los argumentos presentados en otros trabajos relativos a la lectura eventiva de los predicados experimentadores (Arad, 1999; McGinnis, 2001; Pylkkänen, 2002, 2008; Viñas-de-Puig, 2009; Marín y Sánchez Marco, 2012), se propone que la estativa es la lectura que se obtiene por defecto a partir de la Estructura Experimentadora Básica. Asimismo, se propone que las interpretaciones eventivas son el resultado de añadir núcleos funcionales con rasgos eventivos a esta estructura.

Sin embargo, antes de analizar cómo se pueden obtener predicados experimentadores eventivos, en la subsección siguiente se analizó el tipo de estado que se obtiene cuando se tiene una Estructura Experimentadora Básica con verbo ligero.

\subsubsection{El estado en cuestión}

Los estados resultantes de la Estructura Experimentadora Básica pueden analizarse más detenidamente para identificar el tipo de estado al que pertenecen. Marín y Sánchez Marco (2012) argumentan que los VPEO son manifiestamente predicados estativos, sin propiedades télicas ni dinámicas. Evidencia de este fenómeno es el hecho de que estos predicados no resisten la inclusión de sintagmas que delimitan el límite temporal del evento, como la construcción en x minutos o las perífrasis verbales con acabar o terminar. Esto es lo que Marín y Sánchez Marco (2012) demuestran con los verbos preocupar (27a) e interesar (27b), que claramente pertenecen a la categoría VPEO:

(27) (a) *Esta situación ha preocupado a tus padres en cinco minutos.

(Marín y Sánchez Marco, 2012: ej. 3a)

(b) *Esta situación ha terminado de interesar a tus padres.

(Marín y Sánchez Marco, 2012: ej. 4a)

En los dos ejemplos en (27) observamos que es imposible acotar el límite del significado descrito por los predicados, demostrándose así su carácter atélico y no dinámico. Cabe señalar además que tanto preocupar como interesar introducen 
un EXPERIMENTADOR Dativo y, por lo tanto, pertenecen a la misma subclase de predicados experimentadores que los PEVL (7b). Aplicando las mismas pruebas que proponen Marín y Sánchez Marco (2012), se puede comprobar que los PEVL tampoco son posibles con las mismas construcciones que delimitan el evento:

(28) (a) *Esta situación les ha dado miedo en cinco minutos.

(b) ??'Esta situación nos ha terminado de dar asco.

(29) (a) *Aquesta situació els ha fet por en cinc minuts.

'Esta situación les ha dado miedo en cinco minutos.'

(b) ???Aquesta situació ens ha acabat de fer fasstic.

'Esta situación nos ha terminado de dar asco.'

Como es de esperar, se observa también que los PEVL presentan las mismas características referentes al tipo de estado que los demás VPEO. Tal como afirman Marín y Sánchez Marco (2012), además de otros autores, los VPEO se comportan como predicados stage level, ya que pueden ser acotados temporalmente; los VPES (verbos psicológicos de experimentador sujeto, como temer), son predicados individual level, que se mantienen dentro de unos límites temporales indefinidos (cf. Condoravdi, 1992). Así pues, a diferencia de los VPES (30), tanto los VPEO (31) como los PEVL (32) son compatibles en expresiones de cuantificación temporal:

(30) (a) * Cuando teme tus reacciones, se deprime.

(Marín y Sánchez Marco, 2012: ej. 12a)

(b) *Sempre que odia els teus comentaris, s'enfada.

'Siempre que odia tus comentarios, se enfada.'

(31) (a) Cada vez que se enfada, empieza a llorar.

(Marín y Sánchez Marco, 2012: ej. 11b)

(b) Cada cop que s'emprenya, comença a fotre crits.

'Cada vez que se cabrea, empieza a pegar gritos.'

(32) (a) Cuando le da asco algo, lo dice.

(b) Sempre que li fa mal la panxa, es posa de mal humor.

'Cada vez que le entra dolor de estómago, se pone de mal humor.'

Puesto que los PEVL se comportan de forma parecida a los VPEO analizados por Marín y Sánchez Marco (2012) en los demás análisis usados por estos autores para demostrar su estatividad, se concluye que estos predicados son stage level, con acotación temporal, a diferencia de los VPES.

Un último aspecto a señalar en esta subsección hace referencia a otro tipo de subclasificación estativa de los predicados. Fábregas y Marín (2012) analizan los sustantivos estativos prestando atención a si son estados davidsonianos (estados D) o estados kimianos (estados K). Según estos autores, y basándose en biblio- 
grafía reciente, se pueden clasificar los estados más allá de los criterios utilizados para determinar si son stage level o individual level: los estados D son predicados pseudoestativos que permiten modificación relativa al lugar y al modo; por el contrario, los estados $\mathrm{K}$ son estados en los que tal modificación no es posible (Fábregas y Marín, 2012: 44). En su trabajo, Fábregas y Marín (2012) afirman que todos los sustantivos estativos (incluyendo los psicológicos o experimentadores) son estados K, ya que a diferencia de lo que ocurre con los sustantivos dinámicos, son incompatibles con modificadores de lugar (33), con adjetivos de modo (34) o con adjetivos ambiguos que denotan una lectura temporal (35) (en este último caso, sólo se puede obtener una lectura de gradación, no temporal):

(33) el aburrimiento de Juan con la naturaleza (*en un silla)

(34) su (*elegante) aburrimiento

(Fábregas y Marín, 2012: ej. 15a)

(Fábregas y Marín, 2012: ej. 19b)

(35) una preocupación ${ }^{\#}$ reducida

(Fábregas y Marín, 2012: ej. 23b)

Si Fábregas y Marín (2012) estuvieran en lo cierto, lo lógico sería suponer que todas las raíces $V_{\text {Exp-A }}$ que aparecen en los PEVL, al ser sustantivos estativos, son estados K; como tales, estos argumentos no deberían permitir modificación con construcciones de lugar o temporales. Sin embargo, algunos de estos argumentos parecen permitir dichas modificaciones (36), (37), mientras que con otros tal modificación no es posible (38):

(36) (a) dolor en el estómago

(b) mal a la panxa 'dolor en el estómago'

(37) (a) un dolor repentino

(b) un fastic sobtat 'un asco repentino'

(38) (a) ?? una lástima repentina

(b) ??? una angúnia sobtada 'una zozobra repentina'

Visto este comportamiento heterogéneo respecto a las propiedades estativas de los argumentos EXPERIENCIA, en contra de lo propuesto por Fábregas y Marín (2012), se demuestra que estos argumentos no pueden ser todos estados K. Para dar cuenta de esta variación, propongo que estos argumentos, como raíces que se introducen en la estructura sintáctica ya vienen subcategorizados con rasgos eventivos, que son los que determinan si estos argumentos son, en su base, estados D 
o estados K. Un análisis por subcategorización como este nos sirve, además, para analizar el comportamiento también variable en relación con la posibilidad de obtener lecturas eventivas (i.e. dinámicas) de los PEVL.

En relación con lo apuntado en esta subsección, cabe hacer hincapié en un último punto. Fábregas et al. (2012), y en cierto modo Fábregas y Marín (2012), argumentan que los sustantivos psicológicos (o experimentadores) se derivan de verbos, de los que heredan la información eventiva. Sin embargo, en catalán (y es de suponer que también en castellano), existen PEVL en los que la EXPERIENCIA aparece como un argumento independiente, pero nunca como forma verbal (39), (40):

(39) (a) fer mal 'hacer daño, dañar'

(b) *malejar, *malar

(40) (a) fer nosa 'estorbar, molestar'

(b) *nosejar, *nosar

Con estos ejemplos se demuestra que los PEVL siguen la estructura argumen-

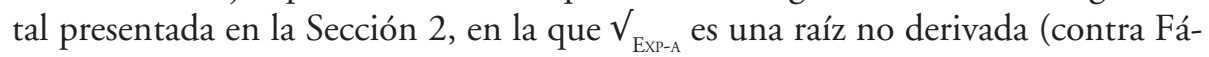
bregas et al., 2012) y que entra en la derivación con rasgos eventivos (y estativos) ya determinados.

A partir del comportamiento de los PEVL en lecturas estativas, y teniendo como base las propiedades ya descritas del argumento EXPERIENCIA, en la subsección siguiente se presenta el análisis que da cuenta de la posibilidad de obtener interpretaciones eventivas (con o sin CAUSANTE externo) con estos predicados.

\subsection{Lecturas eventivas de los predicados experimentadores}

Ya se ha visto que algunos predicados experimentadores, incluyendo los PEVL, permiten interpretaciones tanto estativas como eventivas, como ya han indicado varios autores (Belletti y Rizzi, 1988; Pesetsky, 1995; Arad, 1999; McGinnis, 2001; Pylkkänen, 2002, 2008; Viñas-de-Puig, 2009; entre otros). Dicho contraste se observa en diferentes lenguas, como se comprobó en los ejemplos (22) a (24) repetidos a continuación:

(41) (a) Me asustan los comentarios racistas.

(b) Esos niños me asustaron ayer.

(42) (a) Em fan por els comentaris racistes. 'Me asustan los comentarios racistas.'

(b) Aquells nens em van fer por ahir. 
'Esos niños me asustaron ayer.'

(43) (a) Racist comments scare me.

'Me asustan los comentarios racistas.'

(b) Those children scared me yesterday.

'Esos niños me asustaron ayer.'

Como se puede observar en estos ejemplos, tanto en castellano (41) como en catalán (42) y en inglés (43), algunos predicados experimentadores permiten lecturas estativas y agentivas: en las frases (a) de cada pareja en los ejemplos (41) a (43), la única de las interpretaciones posibles es la de un estado; por el contrario, en las frases (b) la lectura que se obtiene con mayor facilidad es eventiva, con la presencia de un CAUSANTE de la experiencia descrita. Teniendo en cuenta que la ESTRUCTURA EXPERIMENTADORA BÁSICA da como resultado únicamente interpretaciones estativas (Sección 3.1.), y siguiendo postulados recientes sobre la estructura eventiva de los predicados experimentadores (Arad, 1999; McGinnis, 2001; Pylkkänen, 2002, 2008), se propone que las lecturas eventivas son el resultado de añadir información eventiva por encima de $S v_{\mathrm{EXP}}$, de forma similar a lo propuesto por varios autores (Pylkkänen, 1999, 2002, 2008; McGinnis, 2000, 2001; Schäfer, 2009; Marín y McNally, 2009; entre otros). En (44) y (45) se muestran las dos estructuras que dan como resultado predicados experimentadores eventivos:

(44) Predicado experimentador incoativo

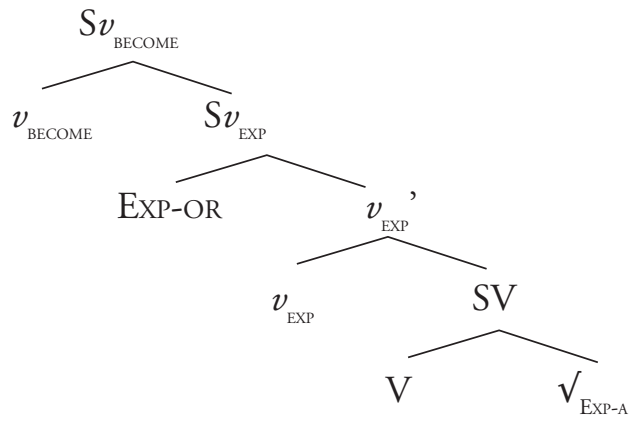

(45) Predicado experimentador causativo

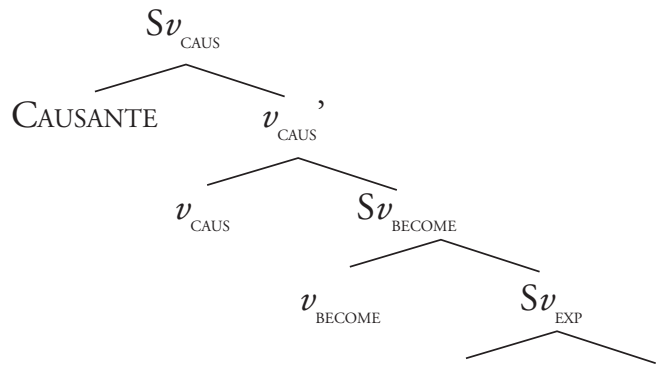


Predicados psicológicos y estructuras con verbo ligero: Del estado al evento / R. VIÑAS-DE-PUIG

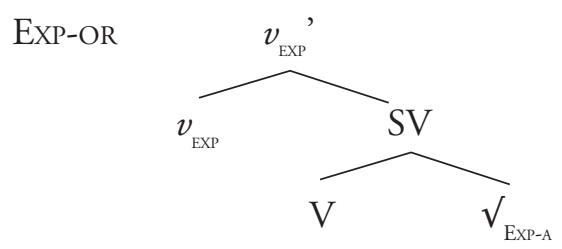

Puesto que las estructuras en (44) y (45) son diferentes, las interpretaciones resultantes también son diferentes. En (44), la proyección funcional $v_{\text {вЕсоме }}$ introduce información eventiva y, puesto que se trata de un núcleo inacusativo (que no introduce un argumento externo), la única interpretación posible es la de un evento incoativo (es decir, una experiencia sin causante externo). La estructura en (45) también introduce información eventiva, ya que incluye el núcleo $v_{\text {вЕсомЕ }}$; sin embargo, a diferencia de la estructura anterior, en esta derivación se une el núcleo $v_{\text {CAUS }}$ a $\mathrm{S} v_{\text {BECOME }}$. El núcleo $v_{\text {CAUS }}$ introduce un CAUSANTE y, por consiguiente, la interpretación que se obtiene es la de un predicado experimentador eventivo con causación externa.

Si se analiza de nuevo las lecturas estativas observadas en la Sección 3.1., podemos comprobar como los PEVL estativos no pueden aparecer con sintagmas temporales que focalicen el límite final del evento, como se comprueba en los ejemplos en catalán que se muestra en (46):

(46) (a) *Em fa mal la mà en cinc minuts. '* Me duele la mano en cinco minutos.'

(b) *En Jordi té gana en cinc minuts. '*Jordi tiene hambre en cinco minutos.'

Asimismo, estos predicados tampoco son modificables con frases adverbiales del tipo a propósito / detenidamente (cf. Dowty, 1979), como se observa en (47):

(47) ??:Em fa mal la mà expressament.

???"Me duele la mano a propósito.'

Por el contrario, si las estructuras presentadas en (44) y (45) son ciertas, sería de esperar que se obtuvieran PEVL que permitan construcciones temporales:

(48) (a) Me salen granos en cinco minutos.

(b) Esa música me dio dolor de cabeza en dos minutos.

(49) (a) Em surten grans en cinc minuts. 'Me salen granos en cinco minutos.'

(b) Aquesta música em fa venir mal de cap en cinc minuts. 'Esta música me da dolor de cabeza en cinco minutos.' 
Tanto en el ejemplo (48) como en el (49), la introducción de una construcción temporal no provoca que la frase sea agramatical, confirmándose así que los PEVL en esos ejemplos son eventos (y no estados). De estos dos ejemplos, cabe notar dos aspectos interesantes: i. en las frases (a) no se observa la presencia de un CAUSANTE externo, mientras que en las frases (b) sí observamos un CAUSANTE (esa música en (48b); aquesta música en (49b)); y ii. observamos una variación en la forma del verbo ligero. El primer hecho es el resultado de tener una derivación con dos estructuras diferentes: las frases en (a) se obtienen a partir de la estructura incoativa (44); las frases en (b) son el resultado de la estructura causativa (45).

En lo referente a la forma fonológica del verbo ligero, de forma parecida a lo que Crous y Gràcia (2013) argumentan, propongo que la variación observada es el resultado de la suma de rasgos eventivos (y semánticos) presentes en los diferentes núcleos funcionales. Es decir, si tenemos una estructura estativa, con únicamente el núcleo $v_{\mathrm{EXP}}$, la forma del verbo ligero será dar en castellano o fer 'hacer' en catalán. Si se une el núcleo $v_{\text {вЕсоме }}$ a $S v_{\text {ЕХP }}$, entonces tendremos que el verbo ligero es salir en castellano o sortir 'salir' en catalán; de forma similar, si se une la proyección $v_{\text {CAus }}$, el resultado es el verbo dar en castellano y la construcción perifrástica fer venir 'lit. hacer venir' en catalán.

En este mismo sentido, y como bien apuntan Crous y Gràcia (2013) en su estudio, la forma del verbo (ligero) depende también del tipo de experiencia que se describe. Por lo tanto, el argumento EXPERIENCIA también debe venir subcategorizado del lexicón con unos rasgos que, al combinarse con los rasgos de las diferentes proyecciones funcionales, da como resultado las diferentes posibles formas fonológicas del verbo ligero.

Si se presta más atención a las lecturas agentivas de los predicados experimentadores, ya mencionadas por varios autores (Belletti y Rizzi, 1988; Van Voorst, 1992; Landau, 2005; entre otros), se observa que también son posibles en lenguas no romances:

(50) Kim bothers me every morning. 'Kim me molesta todas las mañanas.'
(51) Manna
dalâni
yâtamana.
Nom.2PL
dolor-Agr.3SG DAT.1SG-[dar]-Prs.2PL

'Ustedes me están haciendo daño.' (lit. 'Ustedes me están dando dolor.')

Tanto en inglés (50) como en mayangna (51) se observa la posibilidad de obtener predicados experimentadores con lecturas agentivas, al igual que en castellano y catalán. Además, si estos predicados son realmente agentivos, como se propone, entonces sería de esperar que pudieran aparecer con construcciones adverbiales del tipo a propósito / detenidamente (cf. Dowty, 1979). Esto es lo que se demuestra en los ejemplos (52) y (53): 
Predicados psicológicos y estructuras con verbo ligero: Del estado al evento / R. VIN̄AS-DE-PUIG

(52) Los niños lo molestan a propósito.

(53) Aquells nens em fan mal expressament.

'Esos niños me hacen daño a propósito.'

En los dos ejemplos anteriores se observa que es gramatical introducir una expresión con el significado 'a propósito', que solo es posible si tenemos lecturas agentivas. Asimismo, en ambos casos se nota la presencia de un CAUSANTE externo: los niños en (52) y aquells nens 'esos niños' en (53).

Si se toma como válida la estructura causativa presentada en (45), en la que el Causante o Agente (dependiendo de si se trata de un argumento con rasgos [-animado] o [+animado], respectivamente) es el argumento externo, sería de esperar que este argumento tuviera propiedades de un argumento externo verdadero.

(54) $[\text { El teu germà }]_{\mathbf{i}}$ em va fer por per $\mathbf{P R O}_{\mathbf{i}}$ divertir-se $_{\mathbf{i}}$. 'Tu hermano me asustó para divertirse.'

En el ejemplo catalán en (54) se puede comprobar como el SD el teu germà 'tu hermano' es el CAUSANTE del predicado experimentador y, al tratarse de un argumento [+animado], logra el rol de AGENTE. Según la propuesta que se presenta, este argumento se une a la estructura en la posición de especificado del $S v_{\text {Caus' }}$, desde donde puede establecer una relación de ligamiento con el argumento PRO de la cláusula subordinada no finita. Se demuestra así que la estructura experimentadora causativa no sólo es posible, sino que además introduce un argumento Causante/Agente en una posición de argumento externo.

\subsection{Un análisis compuesto}

Hasta este punto se ha tratado la existencia de PEVL con diferentes lecturas (estativa, incoativa y causativa/agentiva) y se ha argumentado que la existencia de las lecturas eventivas es el resultado de unir proyecciones funcionales eventivas $\left(v_{\text {BECOME}}\right.$, $\left.v_{\text {CAUS }}\right)$ al $S v_{\text {EXP }}$ estativo. Sin embargo, este análisis puramente estructural no da cuenta de todas las posibilidades de los PEVL respecto a su interpretación eventiva.

(55) (a) Esos niños me hicieron daño a propósito.

(b) ??Esos niños me dieron asco a propósito.

(c) ???*Esos niños me dieron lástima a propósito

(56) (a) Aquells nens em van fer mal expressament. 'Esos niños me hicieron daño a propósito.'

(b) "'Aquells nens em van fer fastic expressament. 'Esos niños me dieron asco a propósito.' 
(c) ${ }^{m ? / *}$ Aquells nens em van fer angúnia expressament.

'Esos niños me dieron zozobra a propósito.'

De los ejemplos (55) y (56) hay dos hechos a remarcar: i. todas estas frases presentan una lectura causativa/agentiva, como se demuestra con la inclusión de la expresión a propósitolexpressament 'a propósito' (que, como ya se ha mencionado, nos fuerza la presencia de un AGENTE); y ii. se observa una variación en el nivel de gramaticalidad dependiendo del tipo de experiencia que se describe. Si la interpretación causativa/agentiva sólo dependiera de la presencia de una proyección funcional con estos rasgos (como la que se presenta en (45)), se podría suponer entonces que es posible obtener lecturas causativas/agentivas con cualquier tipo de experiencia. Sin embargo, las frases (b) y (c) en (55) y (56) demuestran que no es así.

Para resolver este punto problemático, se presenta un análisis que combina dos aspectos. En primer lugar, y siguiendo lo que se ha afirmado en la Sección 3.2., para obtener lecturas eventivas (incluyendo las causativas/agentivas) es necesario unir a la derivación los núcleos funcionales pertinentes, con rasgos eventivos (es decir, $v_{\text {Bвсоме }}$ lleva rasgos eventivos incoativos, $v_{\text {CAus }}$ lleva rasgos causativos). Y en segundo lugar, siguiendo análisis recientes de subcategorización (Crous y Gràcia, 2013; cf. Paster, 2006, 2009; Lloret y Clua, 2013), el argumento ExpERIENCIA viene especificado del lexicón con rasgos eventivos. Se establece entonces una relación de concordancia de rasgos entre los núcleos funcionales eventivos $\left(v_{\text {BECOME}}\right.$, $\left.v_{\text {CAUS }}\right)$ y el argumento EXPERIENCIA: si hay concordancia entre los rasgos de la EXPERIENCIA y los de los núcleos funcionales, se obtiene una lectura eventiva (55a) (56a); por el contrario, si el argumento EXPERIENCIA carece de rasgos eventivos, no se puede establecer la concordancia con los rasgos de los núcleos funcionales $v_{\text {вEсоме }}$ o $v_{\text {caus }} \mathrm{y}$, como consecuencia, únicamente es posible una interpretación estativa (55b, c) (56b, c).

Este análisis, además, concuerda con otras argumentaciones en la bibliografía relativas a la carga eventiva de raíces verbales (Klein, 1994; entre otros). Según estos análisis, las raíces predicativas (en este caso, la raíz $V_{\text {Exp-A }}$ ) llevan información sobre el Aktionsart del evento y, por lo tanto, sólo son posibles en las lecturas que concuerdan con esta información subcategorizada.

\section{RESUMEN Y CONCLUSIONES}

En este trabajo se propone un análisis que da cuenta de las diferentes lecturas (estativas y eventivas) de los predicados experimentadores con verbo ligero (PEVL) 
usando datos de castellano y catalán, pero también aplicable a otras lenguas. Según esta propuesta, los PEVL son el resultado de tener una Estructura EXPERIMENTADORA BÁsICA, con núcleo $\mathrm{V}_{\mathrm{EXP}}$, que permite dos posibilidades relativas a la expresión del argumento EXPERIENCIA $\left(V_{\text {EXP-A }}\right)$ : en caso de que la raíz $V_{\text {Exp-A }}$ no se incorpore a $\mathrm{V}$, se obtiene entonces un PEVL.

Teniendo en cuenta que la Estructura Experimentadora Básica únicamente puede dar lecturas estativas, las posibles interpretaciones eventivas de los PEVL dependen de dos factores. En primer lugar, es necesaria la unión de la proyección funcional $v_{\text {вЕсомF }}$, que da como resultado una interpretación incoativa (puesto que se trata de un núcleo inacusativo), o la unión de la proyección funcional $v_{\text {caUs }}$ a $S v_{\text {ввсоме }}$, que (ya que esta proyección introduce un CAUSANTE). En segundo lugar, los rasgos de estas proyecciones funcionales eventivas deben establecer una relación de concordancia con la raíz EXPERIENCIA $\left(V_{\text {EXP-A }}\right)$, que viene subcategorizada con rasgos eventivos. En caso de que no haya concordancia entre los rasgos eventivas de las proyecciones funcionales y los de la raíz EXPERIENCIA $\left(V_{\text {EXP-A }}\right)$, no se podrán obtener lecturas eventivas.

Este análisis da cuenta de la variación en la posibilidad de tener (o no) lecturas eventivas dependiendo del tipo de experiencia descrita, además de presentar una explicación plausible de la estructura argumental de estos predicados. Igualmente, complementa otros estudios recientes sobre predicados experimentadores en romance que también se basan en un análisis de subcategorización. Por último, esta propuesta también puede servir de base para demostrar la variación en la forma del verbo ligero en los PEVL.

\subsection{Cuestiones para investigaciones futuras}

Si se toma el análisis que aquí se presenta como válido, aparecen algunas cuestiones que pueden ser interesantes para futuros trabajos de investigación. En la Sección 3 se propone la presencia recursiva del núcleo funcional $v$, aunque con diferentes variaciones (cf. Arad, 1999; McGinnis, 2000). Si, siguiendo trabajos recientes (cf. Chomsky, 2001; Gallego, 2010), se considera que Sv constituye una Fase, entonces cabe preguntarse cuántas Fases aparecen si se tiene un predicado experimentador causativo, en el que se introducen tres variaciones de $v$ (i.e. $v_{\mathrm{EXP}}$, $v_{\text {вЕсоме }}$ y $v_{\text {CAUS }}$ ). Esta cuestión, además, está relacionada con la asignación de Caso estructural, ya que algunos autores (Brattico, 2008; entre otros) argumentan que la asignación de Caso es la última operación que se da antes de cerrar una Fase. Todas estas cuestiones, a pesar de su evidente interés, no son pertinentes al tema específico de este trabajo y, por lo tanto, se dejan para investigaciones futuras. 
RLA. Revista de Lingüística Teórica y Aplicada, 52 (2), II Sem. 2014

\section{REFERENCIAS}

Adger, David y Ramchand, Gillian. (2006). Psych nouns and predication. En Christopher Davis, Amy Rose Deal \& Youri Zabbal. (eds.), Proceedings of NELS 36 (pp. 89-102). Amherst, MA, Estados Unidos: GLSA, UMass.

Arad, Maya. (1998). Psych-notes. UCL Working Papers in Linguistics, 10, 1-22.

Arad, Maya. (1999). What counts as a class? The case of psych verbs. MIT Working Papers in Linguistics, 35, 1-23.

Belletti, Adriana y Rizzi, Luigi. (1988). Psych-verbs and Theta-Theory. Natural Language and Linguistic Theory, 6, 291-352.

Bouchard, Denis. (1992). Psych constructions and conceptual structures. En Hirschbühler, Paul y Konrad Koerner (eds.), Romance Languages and Modern Linguistic Theory (pp. 25-44). Philadephia, PA, Estados Unidos: John Benjamins.

Brattico, Pauli. (2008). Kayne's Theory of case and finnish DPs. Nordic Journal of Linguistics, 31, 5-44.

Charles, Jacinto y Torrez, Mateo. (2008). Morfología y sintagma nominal de los verbos impersonales en gramática de la lengua sumu-mayangna. Monografía de Licenciatura. Universidad de las Regiones Autónomas de la Costa Caribe Nicaragüense (URACCAN).

Chomsky, Noam. (2001). Derivation by Phase. En Kenstowicz, Michael (ed.), Ken Hale. A life in language. Cambridge, MA, Estados Unidos: MIT Press. $1-52$.

Condoravdi, Cleo. (1992). Individual-level predicates in conditional clauses. En LSA Annual Meeting. Philadelphia, PA, Estados Unidos.

Croft, William. (1993). Case marking and the semantics of mental verbs. En James Pustejovsky (ed.), Semantics and the lexicon (pp. 55-72). Dordrecht, Holanda: Kluwer.

Crous, Berta y Gràcia, Lluïsa. (2013). Movimientos morbosos: De la categorización de las afecciones a la selección del verbo. Girona, Catalunya, España: Ms, Universitat de Girona.

Cuervo, María Cristina. (2003). Really affected arguments: Datives in causatives and inchoatives and other bi-eventive configurations. Cambridge, MA, Estados Unidos: MIT Ling Lunch.

Cuervo, María Cristina. (2010). Against ditransitivity. Probus, 22 (2), 151-180.

Dowty, David. (1979). Word meaning and Montague grammar. Dordrecht, Holanda: Reidel.

Fábregas, Antonio y Marín, Rafael. (2012). State nouns are Kimian states. Romance Languages and Linguistic Theory 2010: Selected Papers from 'Going Romance' Leiden 2010. 4 (pp. 41-64). Amsterdam, Holanda: John Benjamins.

Fábregas, Antonio; Marín, Rafael y McNally, Louise. (2012). From psych verbs to 
nouns. Telicity, change, and state: A cross-categorial view of Event structure (pp. 162-185). Oxford, Inglaterra: Oxford University Press.

Franco, Jon y Huidobro, Susana. (2003). Psych Verbs in Spanish Leísta Dialects. En: Montrul, Silvina y Ordóñez, Francisco (Eds.). Linguistic Theory and Language Development in Hispanic Languages (pp. 138-157). Somerville, MA, Estados Unidos: Cascadilla Press.

Gallego, Ángel J. (2010). Phase theory. Amsterdam, Holanda: John Benjamins.

Hale, Kenneth y Keyser, Samuel Jay. (1993). On argument structure and the lexical expression of syntactic relations. En Kenneth Hale y Samuel Jay Keyser (eds.), The View from Building 20: Essays in Linguistics in Honor of Sylvain Bromberger (pp. 53-109). Cambridge, MA, Estados Unidos: MIT Press.

Hale, Kenneth y Keyser, Samuel Jay. (2002). Prolegomenon to a Theory of argument structure. Cambridge, MA, Estados Unidos: MIT Press.

Haspelmath, Martin y Caruana, Sandro. (2000). Subject diffuseness in Maltese: On some subject properties of experiential verbs. Folia Lingüistica, 24, 245265.

Klein, Wolfgang. (1994). Time in language. Londres, Inglaterra: Routledge.

Kratzer, Angelika. (1996). Severing the external argument from the verb. En Rooryck, Johann \& Laurie Zaring (eds.), Phrase structure and the lexicon (pp. 109-137). Dordrecht, Holanda: Kluwer.

Landau, Idan. (2005). The locative syntax of experiencers. Be'er Sheva, Israel: Ms, Ben Gurion University.

Lloret, María Rosa y Clua, Esteve. (2013). Al-lomorfia externa en la flexió verbal del català des de la perspectiva de la teoria de l'optimitat. 2013 North American Catalan Society (NACS) Symposium. Toronto, ON, Canadá.

Marín, Rafael y McNally, Louise. (2009). Inchoativity, change of state, and telicity: Evidence from Spanish reflexive psychological verbs. Lille, Francia; Barcelona, Catalunya, España: Ms. CNRS/Université de Lille and Universitat Pompeu Fabra.

Marín, Rafael y Sánchez Marco, Cristina. (2012). Verbos y nombres psicológicos: juntos y revueltos. Borealis-An International Journal of Hispanic Linguistics, 1 (2), 91-108.

Mateu, Jaume. (2012). Conflation and incorporation processes in resultative constructions. Telicity, change, and state: A cross-categorial view of Event structure (pp. 252-278). Oxford, Inglaterra: Oxford University Press.

Masullo, Pascual. (1992). Incorporation and case theory in Spanish. A crosslinguistic perspective. Tesis doctoral. University of Washington.

McGinnis, Martha. (2000). Event heads and the distribution of Psych-roots. En Alexander Williams \& Elsi Kaiser (eds.), Current work in Linguistics. Penn Working Papers in Linguistics, 6(3), 107-144.

McGinnis, Martha. (2001). Semantic and morphological restrictions in experien- 
cer predicates. En John T. Jensen \& Gerard Van Herk (eds.), Proceedings of the 2000 CLA Annual Conference (pp. 245-256). Ottawa, Canada: Cahiers Linguistiques d'Ottawa.

Paster, Mary. (2006). Phonological conditions on affixation. Tesis doctoral. University of California, Berkeley.

Paster, Mary. (2009). Explaining phonological conditions on affixation: Evidence from suppletive allomorphy and affix ordering. Word Structure, 2 (1), 18-37.

Pesetsky, David. (1987). Binding problems with experiencer verbs. Linguistic Inquiry, 18, 126-40.

Pesetsky, David. (1995). Zero Syntax: Experiencers and Cascades. Cambridge, MA, Estados Unidos: MIT Press.

Pylkkänen, Liina. (1999). External arguments and causation. MIT Working Papers in Linguistics, 35, 161-183.

Pylkkänen, Liina. (2002). Introducing arguments. Tesis doctoral. MIT.

Pylkkänen, Liina. (2008). Introducing arguments. Cambridge, MA, Estados Unidos: MIT Press.

Ramchand, Gillian. (2008). Verb meaning and the lexicon: A First Phase Syntax. New York, NY, Estados Unidos: Cambridge University Press.

Rigau, Gemma. (1990). Les propietats d'agradar: Estructura temàtica i comportament sintàctic. Caplletra, 8, 7-20.

Rosselló, Joana. (2002). El SV, I: Verb i arguments verbals. En Joan Solà, Maria Rosa Lloret, Joan Mascaró \& Manuel Pérez Saldanya (eds.), Gramàtica del català contemporani. Vol 2. (pp. 1853-1949). Barcelona, Catalunya, España: Empúries.

Schäfer, Florian. (2009). Two types of external argument licensing - The case of causers. Stuttgart, Alemania: Ms. Universität Stuttgart.

Torrego, Esther. (1996). Experiencers and raising verbs. En Robert Freidin (ed.), Current Issues in Comparative Grammar (pp. 101-120). Dordrecht, Holanda: Kluwer.

Van Voorst, Jan. (1992). The aspectual semantics of psychological verbs. Linguistics and Philosophy, 15(1), 65-92.

Viñas-de-Puig, Ricard. (2009). The argument of experience. Experience predicates and argument structure in Catalan and Mayangna. Tesis doctoral. Purdue University. 